\title{
Endoscopic ultrasound in the pre-therapeutic staging of gastroesophageal adenocarcinoma: the diagnostic value in defining patients eligible for a neoadjuvant chemotherapy regimen
}

\author{
Wilko Staiger ${ }^{1 *}$, Ulrich Ronellenfitsch ${ }^{1 *}$, Ralf-Dieter Hofheinz ${ }^{2}$, Philipp Ströbel ${ }^{3}$, Miriam Hahn ${ }^{1}$, Stefan Post ${ }^{1}$, Peter Collet ${ }^{1}$, \\ Georg Kähler', Matthias Schwarzbach ${ }^{1}$ \\ 1Department of Surgery, University Hospital Mannheim, Ruprecht-Karls-University Heidelberg, Mannheim, Germany \\ 2Medical Oncology, University Hospital Mannheim, Ruprecht-Karls-University Heidelberg, Mannheim, Germany \\ 3Institute of Pathology, University Hospital Mannheim, Ruprecht-Karls-University Heidelberg, Mannheim, Germany \\ *These authors contributed equally.
}

Videosurgery and other miniinvasive techniques 2010; 5 (1): 1-6 DOI: $10.5114 /$ wiitm.2010.13599

\begin{abstract}
Introduction: Neoadjuvant chemotherapy regimens have been proven to decrease tumour size and stage and significantly improve progression-free and overall survival in the treatment of locoregional advanced gastroesophageal adenocarcinomas. Therefore, the pre-therapeutic staging of the tumour extension is of utmost importance for stratification of patients into this individualized therapy regimen. Within the last years most experience has been gained using endoscopic ultrasound (EUS) and it has been considered as a valuable tool for the assessment of locoregional disease.

Aim: To evaluate the accuracy of endoscopic ultrasound for the preoperative staging of locally advanced gastroesophageal adenocarcinomas with regard to defining patients' eligibility for neoadjuvant chemotherapy.

Material and methods: Between January 2006 and June 2007 consecutive patients $(n=47)$ who underwent resection of a gastroesophageal adenocarcinoma and would have been potentially eligible for neoadjuvant chemotherapy were included in this study. Preoperative EUS staging was compared to the postoperative histopathology results. Furthermore, the specificity, sensitivity and positive and negative predictive values for serosal invasion and/or lymph node positivity as an eligibility criterion for neoadjuvant chemotherapy were evaluated.

Results: Thirty-seven patients were included in the analysis with adenocarcinomas of the distal oesophagus $(n=7)$, the oesophagogastric junction $(n=8)$ and the stomach $(n=22)$. The overall accuracy for predicting the $T$ stage was $64.9 \%(n=24)$ with an over- and understaging of $13.5 \%(n=5)$ and $21.6 \%(n=8)$, respectively. The overall accuracy for predicting the $N$ stage was $64.7 \%(n=22)$, with an over- and understaging of $26.5 \%(n=9)$ and $8.8 \%(n=3)$. Twenty-five out of 37 patients would have met the eligibility criteria for enrolment in a neoadjuvant chemotherapy regimen. The sensitivity was $76 \%(n=19 / 25)$. The specificity was $75 \%(n=9 / 12)$. The positive predictive value was $86.4 \%$. The negative predictive value was $60 \%$.

Conclusions: In this series the sensitivity and specificity of EUS for identification of patients eligible for neoadjuvant chemotherapy seem acceptable. Especially the positive predictive value, which expresses the probability that a patient identified by EUS as eligible for neoadjuvant chemotherapy met the corresponding histopathological criteria, was high (86.4\%). Therefore, it seems justified to apply neoadjuvant chemotherapy in patients identified as eligible by EUS. This

Address for correspondence:

Dr. Wilko Staiger, Department of Surgery, University Hospital Mannheim, 68135 Mannheim, Germany, phone: 0049-621-383-2555, fax: 0049-621-383-2166, e-mail: wilko.staiger@hotmail.de
\end{abstract}


underpins the importance of EUS in the staging of gastroesophageal carcinoma. In patients with a "negative" EUS result, the staging needs to be complemented by additional diagnostic modalities such as CT, PET or laparoscopy to facilitate the correct identification of patients who meet the histopathological inclusion criteria for neoadjuvant chemotherapy.

Key words: endosonography, stomach neoplasm, esophageal neoplasms, neoadjuvant chemotherapy, neoplasm staging.

\section{Introduction}

In spite of a steady decline over the last decades, gastroesophageal adenocarcinomas are still the fourth most common cancer and the second most common cause of cancer death worldwide, with more than 900,000 new cases and 700,000 deaths per year [1, 2]. In the absence of metastatic disease, surgical resection with negative microscopic margins is the only potentially curative treatment. Whereas patients with early-stage tumours have a 5 -year survival rate of over $90 \%$ [3], recurrence compromises about $80 \%$ of patients with locally advanced disease [4]. Consequently, for these tumour stages 5-year survival rates after primary surgery without multimodal therapy do not exceed $25-30 \%$ [5]. Recently, two randomised controlled trials have demonstrated that perioperative chemotherapy consisting of a neoadjuvant and postoperative cisplatin- and 5-FU based treatment regimen significantly improves progression-free and overall survival in patients with advanced gastric or lower oesophageal adenocarcinoma. The neoadjuvant chemotherapy proved to decrease tumour size and stage $[6,7]$. The improvement of prognosis is most likely due to improved resectability following the downstaging of the tumour, the elimination of micrometastases, and the improvement of tumour-related symptoms. Commonly, patients with UICC stages II and III (UICC 2003), i.e. patients in whom the tumour shows serosa invasion and/or lymph node involvement, are considered eligible for neoadjuvant treatment. In order to identify these patients reliable staging is of utmost importance [8].

Endoscopic ultrasound (EUS) has been considered as a valuable tool for the assessment of tumour invasion and locoregional lymph node status [9]. The diagnostic accuracy in $\mathrm{T}$ (tumour) staging and the specificity for assessing the serosal involvement of tumours shows comparable results to multidetector row computed tomography or magnetic resonance imaging [10]. However, due to technical particularities and inter- and intra-observer variability [11], the value and accuracy of EUS for the pre-therapeutic stratifica- tion of gastroesophageal adenocarcinoma remains controversial. Reliable prediction of tumour extension often depends on histopathological features, tumour size and the localization of tumours [12]. Recent studies demonstrated a lower than expected concordance between EUS and histopathology results, ranging for all T (tumour) stages combined from 35 to $80 \%$ and for $\mathrm{N}$ (nodal) stages from 42 to $78 \%$ [13-15].

\section{Aim}

This study presents our experience with EUS in the pre-therapeutic staging of gastroesophageal adenocarcinomas with the specific aim of assessing the diagnostic value of EUS for defining patients eligible for neoadjuvant chemotherapy.

\section{Material and methods}

Consecutive patients $(n=47)$ who underwent elective resection with curative intention for primary adenocarcinoma of the stomach, gastroesophageal junction and lower oesophagus at our department between January 2006 and June 2007 and who would have been eligible for neoadjuvant chemotherapy based on performance status and co-morbidities were included in this study. In June 2007 neoadjuvant chemotherapy became the consented standard therapy for locoregionally advanced disease at our institution. Single patients who had received neoadjuvant therapy before that date as an individual treatment decision were excluded from the analysis.

Endoscopic ultrasound was carried out at the Endoscopic Unit of the Department of Surgery. If biopsies of the lesion were still needed to confirm the diagnosis, they were done after EUS to avoid falsification of staging results. EUS was performed by using a rotating sector scan echoendoscope (Olympus GIF-UM20; 7.5-12 MHz) after filling the stomach with approximately $600 \mathrm{ml}$ of water. The assessment of the ultrasound images was carried out at the time of the procedure by only two surgical endoscopists (PC, GK). Local tumour infiltration was determined according to the five-layer structure of the gastric 
wall and classified by using the TNM system ( $6^{\text {th }}$ edition, 2003) as recommended by the UICC and expressed by the values uT1-uT4. Lymph nodes were classified as metastatic ( $\mathrm{uN}+$, uNpositive) in accordance with generally accepted criteria or as without metastasis (uNO, uNnegative) [16, 17]. Besides EUS, hydro-CT of the stomach, abdominal ultrasound, and a chest radiograph were additionally used in order to exclude disseminated disease (stage IV disease with peritoneal or haematogenous tumour spread). Surgical treatment for all patients was subtotal or total gastrectomy with D2-lymphadenectomy, transhiatal extended total gastrectomy or abdomino-thoracic resection of the oesophagus.

The results of the EUS staging were compared with histopathological results obtained from the surgical specimen, which were considered "gold standard". The pathological assessment was performed using the standard TNM classification system (UICC TNM Atlas, 6th edition 2002). Pathologists were unaware of EUS results at the time of performing the histopathological examination. For analysis and comparability with appropriate literature, the endosonographic UT2a category was considered as T2 whereas the $u T 2 b$ category was included in the T3 group. Furthermore, pN1-3 stage was considered as one category, $\mathrm{pN}+$ (pNpositive).

$\mathrm{T}$ and $\mathrm{N}$ stage concordance between EUS and pathological results were assessed. The percentage of cases for which EUS yielded under- and overstaging were calculated for each $\mathrm{T}$ and $\mathrm{N}$ category. Furthermore, regarding the accuracy of EUS as a method to decide on enrolment of patients in a neoadjuvant chemotherapy regimen, the sensitivity, i.e. the probability of correctly detecting patients eligible for neoadjuvant chemotherapy with EUS, and specificity, i.e. the probability of correctly detecting patients not eligible for neoadjuvant chemotherapy with EUS, were calculated. In addition, we determined the positive predictive value as the probability that a patient who was considered eligible for neoadjuvant chemotherapy based on EUS indeed showed histopathological serosal or lymph node invasion, and the negative predictive value as the probability that a patient who was not considered eligible for neoadjuvant chemotherapy based on EUS indeed showed no histopathological serosal or lymph node invasion. In the analyses, patients were hypothetically considered eligible for neoadjuvant therapy if the histopathological tumour stage was pT2b to pT4 and/or pN+ (pNpositive).

\section{Results}

In 37 of 47 patients (78.7\%) EUS evaluation was possible (12 women, 25 men, median age 70.5 , range 35.2-86 years). In 2 of 47 patients (4.3\%), EUS was performed but meaningful T staging was not possible due to tumour stenosis. In 8/47 patients (17.0\%) EUS was not performed because of logistic difficulties or patients' wish to undergo surgery regardless of any staging results.

Tumours were located in the distal oesophagus $(n=7 ; 19 \%)$, the gastroesophageal junction $(n=8$; $22 \%)$ and the stomach ( $n=22 ; 59 \%)$, respectively. Tables I and II show the distribution of UT and UN staging results and the corresponding histopathological stage. There were no UT4 or PT4 tumours in this study population.

Tables III and IV show the accuracy of EUS staging for the single UT and UN categories in terms of correct staging, understaging and overstaging as compared with the histopathological staging.

The overall accuracy for correctly predicting the $\mathrm{pT}$ stage with EUS was $64.9 \%$. Preoperative overstaging of the pathological T stage was seen more frequently $(21.6 \%)$ than understaging (13.5\%). The overall accuracy for correctly predicting the pN stage was $64.7 \%$. The accuracy for rightly determining nodal positivity with EUS was $50 \%$, i.e. understaging of the preoperative $\mathrm{N}$ stage was seen in $50 \%$ of cases.

Table I. Individual T stage by EUS (UT) and corre-

lation with pathology results (pT)

\begin{tabular}{|lccccc|}
\hline Variable & pT1 & pT2 & pT3 & pT4 & All cases \\
\hline uT1 & 7 & 5 & - & - & 12 \\
\hline uT2 & 2 & 8 & 3 & - & 13 \\
\hline UT3 & - & 3 & 9 & - & 12 \\
\hline UT4 & - & - & - & - & 0 \\
\hline All cases & 9 & 16 & 12 & 0 & 37 \\
\hline
\end{tabular}

Table II. Individual N stage by EUS ( $\mathrm{UN}$ ) and correlation with pathology results $(\mathrm{pN})$

\begin{tabular}{|lccc|}
\hline Variable & pNO & pN+ & All cases \\
\hline uNO & 13 & 9 & 22 \\
\hline uN+ & 3 & 9 & 12 \\
\hline All cases & 16 & 18 & 34 \\
\hline
\end{tabular}


Table III. Accuracy of the individual T stage with EUS (UT)

\begin{tabular}{|lcccc|}
\hline $\begin{array}{l}\text { EUS } \\
\text { Stage }\end{array}$ & $n$ & $\begin{array}{c}\text { Correct } \\
n(\%)\end{array}$ & $\begin{array}{c}\text { Understaged } \\
n(\%)\end{array}$ & $\begin{array}{c}\text { Overstaged } \\
n(\%)\end{array}$ \\
\hline UT1 & 12 & $7(58.3)$ & - & $5(41.7)$ \\
\hline UT2 & 13 & $8(61.5)$ & $2(15.4)$ & $3(23.1)$ \\
\hline UT3 & 12 & $9(75)$ & $3(25)$ & - \\
\hline UT4 & - & - & - & - \\
\hline All cases & 37 & $24(64.9)$ & $5(13.5)$ & $8(21.6)$ \\
\hline
\end{tabular}

Table IV. Accuracy of the $\mathrm{N}$ stage with EUS (uNO vs. uN+)

\begin{tabular}{|lcccc|}
\hline $\begin{array}{l}\text { EUS } \\
\text { Stage }\end{array}$ & $n$ & $\begin{array}{c}\text { Correct } \\
n(\%)\end{array}$ & $\begin{array}{c}\text { Understaged } \\
n(\%)\end{array}$ & $\begin{array}{c}\text { Overstaged } \\
n(\%)\end{array}$ \\
\hline uNO & 16 & $13(81.3)$ & - & $3(18.7)$ \\
\hline uN+ & 18 & $9(50)$ & $9(50)$ & - \\
\hline All cases & 34 & $22(64.7)$ & $9(26.5)$ & $3(8.8)$ \\
\hline
\end{tabular}

In this study population 25 out of 37 patients would have met the eligibility criteria for enrolment in a neoadjuvant chemotherapy protocol based on postoperative histopathological results ( $T$ stage $\geq \mathrm{p} 2 \mathrm{~b}$ and/or pNpositive). The sensitivity was $76 \%$ $(n=19 / 25)$. The specificity was $75 \%(n=9 / 12)$. The positive predictive value was $86.4 \%$. The negative predictive value was $60 \%$.

\section{Discussion}

The overall mortality of gastroesophageal cancer remains substantial. Patients with locally advanced gastroesophageal cancer, who are at significant risk for locoregional tumour recurrence, benefit from perioperative chemotherapy including a neoadjuvant approach. This treatment modality has shown to decrease tumour size and stage and significantly improve progression-free and overall survival. However, neoadjuvant chemotherapy regimens are associated with toxicity and morbidity, and unnecessary treatment should be minimized. Therefore, a reliable pre-therapeutic staging tool for the selection of patients with locoregional advanced tumours is crucial. Our study specifically assessed the diagnostic value of EUS for determining patients' eligibility for neoadjuvant chemotherapy.
Our study showed that TN staging with EUS is feasible in the vast majority of patients with gastroesophageal adenocarcinoma potentially eligible for neoadjuvant chemotherapy. Only in a small minority was $\mathrm{T}$ and/or $\mathrm{N}$ staging not possible because of tumour stenosis. In our series, a number of patients potentially eligible for neoadjuvant chemotherapy did not undergo EUS because of logistic problems. However, we do not believe that there was a systematic selection of patients who did not undergo EUS and thus it is rather unlikely that our results were biased.

The overall concordance of our EUS results with the individual postoperative pathological $\mathrm{T}$ and $\mathrm{N}$ stage were 64.9 and $64.7 \%$, respectively. For the locoregional staging of disease our findings correspond to the most recent studies, which could not replicate the excellent results described in the past [18].

Furthermore, our results emphasize the difficulty in differentiating subserosal from serosal invasion (T2 vs. T3 stage) of a tumour with EUS. In some areas of the stomach and the gastroesophageal junction, the serosa is very fine and therefore even postoperatively it remains sometimes difficult to distinguish between stages pT2 and $\geq$ pT3. In addition, at the lesser curvature of the stomach there is no serosal layer of the gastric wall and tumours can penetrate directly into the perigastral fatty tissue. In the postoperative histopathological examination, this is often classified as stage pT3, whereas in EUS this is technically stage UT2. For T2 stages, we have seen an EUS accuracy rate of $61.5 \%$, with under- and overestimation of 15.4 and $23.1 \%$. The accuracy rate for determining stage pT3 was $75 \%$, with $25 \%$ underestimation. The accuracy rate of the assessment of lymph node invasion was also low, with $50 \%$ of nodal positive cases not detected. Thus, the value of EUS in the evaluation of regional lymph node metastasis must be considered questionable.

The main focus of the study was, however, not to evaluate the absolute accuracy of EUS in determining the correct preoperative tumour stage but the reliability of EUS in identifying patients eligible for enrolment in a neoadjuvant chemotherapy protocol. In this regard, it is crucial that EUS correctly identifies patients with stages $\geq \mathrm{pT} 3$ and/or $\mathrm{pN}+$. In this series, the sensitivity, or, in other words, the probability that patients eligible for neoadjuvant chemotherapy were correctly identified by EUS, and the specificity, i.e. the probability that a patient who was not eligible for neoadjuvant chemotherapy was identified as such by 
EUS, were both about 76\%, which seems acceptable. The positive predictive value, which expresses the probability that a patient identified by EUS as eligible for neoadjuvant chemotherapy met the corresponding histopathological criteria, was $86.4 \%$. It would be desirable to achieve a higher positive prediction for eligibility for a neoadjuvant chemotherapeutic regimen in order to spare patients from undergoing unnecessary chemotherapy. To our knowledge, however, there are no studies assessing which positive predictive values can be achieved with CT or MRI or a multimodal approach in this specific setting. The single modalities do have a similar accuracy for predicting $\mathrm{T}$ and $\mathrm{N}$ stages $[19,20]$, but they have not been evaluated as tools for deciding whether patients should receive neoadjuvant chemotherapy. Compared to other commonly used diagnostic procedures such as ultrasound, CT and MRI for the detection of colorectal liver metastases [21, 22], pap smear for the detection of cervical intraepithelial neoplasia [23] or troponin serology for the detection of myocardial ischaemia [24], the positive predictive value which EUS yielded in our study seems reasonably high. In conclusion, it seems justified to apply neoadjuvant chemotherapy in patients with gastroesophageal adenocarcinoma identified by EUS as eligible because only relatively few patients will be unnecessarily treated and because so far there is no evidence that other diagnostic modalities can identify eligible patients more accurately. This underpins the importance of EUS in the pre-therapeutic staging of gastroesophageal carcinoma.

In turn, the negative predictive value, i.e., the probability that a patient identified as not eligible for neoadjuvant treatment by EUS indeed has no tumour invasion into the serosa or lymph nodes, is only $60 \%$, and thus much lower. These results are in accordance with the findings of other authors who have evaluated the value of EUS in the staging of rectal cancer over a 10-year period [25]. They concluded that even though the local tumour staging is underachieved by EUS, the method is still the most accurate staging modality for rectal tumours and allows adequate selection of patients for different therapeutic regimens.

In patients with a "negative" EUS result, the staging needs to be complemented by additional diagnostic modalities such as CT or PET to facilitate the correct identification of patients who meet the inclusion criteria for neoadjuvant chemotherapy. Besides these non-invasive measures, staging laparoscopy seems to be a valuable option in detecting nodal-positive disease and tumours with a deep wall invasion which had remained undiagnosed in imaging procedures [26]. It should also be used in any case of suspected peritoneal metastases to rule out or confirm implants which would preclude a curative approach and thus neoadjuvant treatment.

\section{Conclusions}

In summary, our study was able to show that EUS is a feasible and effective staging tool for patients with gastroesophageal adenocarcinoma potentially eligible for neoadjuvant chemotherapy. The exam is able to assess $\mathrm{T}$ and $\mathrm{N}$ stage in the vast majority of patients. Its positive predictive value for determining locally advanced growth or nodal spread is reasonably high and therefore tumours identified as such by EUS should be treated with neoadjuvant chemotherapy. Because of a lower negative predictive value, tumours identified as strictly confined to the inner layers of the gastroesophageal wall should be reconsidered for additional assessment by cross-sectional imaging, PET or staging laparoscopy.

\section{References}

1. Forman D, Burley VJ. Gastric cancer: global pattern of the disease and an overview of environmental risk factors. Best Pract Res Clin Gastroenterol 2006; 20: 633-49.

2. Crew KD, Neugut Al. Epidemiology of gastric cancer. World J Gastroenterol 2006; 12: 354-62.

3. Borie F, Rigau V, Fingerhut A, Millat B. French Association for Surgical Research. Prognostic factors for early gastric cancer in France: Cox regression analysis of 332 cases. World J Surg 2004; 28: 686-91.

4. Wang SJ, Emery R, Fuller CD, et al. Conditional survival in gastric cancer: a SEER database analysis. Gastric Cancer 2007; 10: 153-8.

5. DeMeester SR. Adenocarcinoma of the esophagus and cardia: a review of the disease and its treatment. Ann Surg Oncol 2006; 13: 12-30.

6. Cunningham D, Allum WH, Stenning SP, et al. MAGIC Trial Participants. Perioperative chemotherapy versus surgery alone for resectable gastroesophageal cancer. N Engl J Med 2006; 355: $11-20$.

7. Boige V, Pignon J, Saint-Aubert B, et al. Final results of a randomized trial comparing preoperative 5-fluorouracil (F)/cisplatin $(\mathrm{P})$ to surgery alone in adenocarcinoma of stomach and lower esophagus (ASLE): FNLCC ACCORD07-FFCD 9703 trial. ASCO Annual Meeting Proceedings Part I. 2007; 25 18S: 4510.

8. Chua YJ, Cunningham D. The UK NCRI MAGIC trial of perioperative chemotherapy in resectable gastric cancer: implications for clinical practice. Ann Surg Oncol 2007; 14: 2687-90. 
9. Jones DB. Role of endoscopic ultrasound in staging upper gastrointestinal cancers. ANZ J Surg 2007; 77: 166-72.

10. Kwee RM, Kwee TC. Imaging in local staging of gastric cancer: a systematic rewiew. J Clin Oncol 2007; 25: 2107-16.

11. Meining A, Roesch T, Wolf A, et al. High interobserver variability in endosonographic staging of upper gastrointestinal cancers. Z Gastroenterol 2003; 41: 391-4.

12. Heeren PA, van Westreenen HL, Geersing GJ, et al. Influence of tumor characteristics on the accuracy of endoscopic ultrasonography in staging cancer of the esophagus and esophagogastric junction. Endoscopy 2004; 36: 966-71.

13. Barbour AP, Rizk NP, Gerdes H, et al. Endoscopic ultrasound predicts outcomes for patients with adenocarcinoma of the gastroesophageal junction. J Am Coll Surg 2007; 205: 593-601.

14. Bentrem D, Gerdes H, Tang L, et al. Clinical correlation of endoscopic ultrasonography with pathologic stage and outcome in patients undergoing curative resection for gastric cancer. Ann Surg Oncol 2007; 14: 1853-9.

15. Ganpathi IS, So JB, Ho KY. Endoscopic ultrasonography for gastric cancer: does it influence treatment? Surg Endosc 2006; 20: 559-62.

16. Kimmey MB, Martin RW, Haggitt RC, et al. Histologic correlates of gastrointestinal ultrasound images. Gastroenterology 1989; 96: 433-41.

17. Tio TL, Kallimanis GE. Endoscopic ultrasonography of perigastrointestinal lymph nodes. Endoscopy 1994; 26: 776-9.

18. Roesch T. Endosonographic staging of gastric cancer: a review of literature results. Gastrointest Endosc Clin N Am 1995; 5: 549-57.

19. Habermann CR, Weiss F, Riecken R, et al. Preoperative staging of gastric adenocarcinoma: comparison of helical CT and endoscopic US. Radiology 2004; 230: 465-71.

20. Kim AY, Han JK, Seong CK, et al. MRI in staging advanced gastric cancer: is it useful compared with spiral CT? J Comput Assist Tomogr 2000; 24: 389-94.

21. Bhattacharjya S, Bhattacharjya T, Baber S, et al. Prospective study of contrast-enhanced computed tomography, computed tomography during arterioportography, and magnetic resonance imaging for staging colorectal liver metastases for liver resection. Br J Surg 2004; 91: 1361-9.

22. Larsen LP, Rosenkilde M, Christensen H, et al. The value of contrast enhanced ultrasonography in detection of liver metastases from colorectal cancer: a prospective double-blinded study. Eur J Radiol 2007; 62: 302-7.

23. Ronco G, Cuzick J, Pierotti P, et al. Accuracy of liquid based versus conventional cytology: overall results of new technologies for cervical cancer screening: randomised controlled trial. BMJ 2007; 335: 28.

24. Bucciarelli-Ducci C, Rasile C, Proietti P, et al. Troponin I as a specific marker of myocardial injury: from theory to clinical practice in the diagnosis of acute coronary syndrome. Coron Artery Dis 2004; 15: 499-504.

25. Knaebel HP, Koch M, Feise T, et al. Diagnostics of rectal cancer: endorectal ultrasound. Recent Results Cancer Res 2005; 165: 46-57.

26. D'Ugo DM, Persiani R, Caracciolo F, et al. Selection of locally advanced gastric carcinoma by preoperative staging laparoscopy. Surg Endosc 1997; 11: 1159-62. 